\title{
Logical Paradoxes in Non-Classical Logic Systems
}

\author{
Serge Dolgikh [0000-0001-5929-8954] \\ Dept. of Information Technology, \\ National Aviation University
}

\begin{abstract}
It is shown that well-known logical paradoxes such as Barber paradox can be interpreted differently in non-classical logic systems such as multivalued, continuous and quantum logic with possibility of solutions of the paradox. The results of this research can have applications in investigations of completeness of logic systems.
\end{abstract}

Keywords: Fuzzy logic, multi-valued logic, logic systems, logical paradoxes.

\section{Introduction}

Non-classical logic systems, that is, those in which a logical variable can have more than two values have been researched in depth over the decades producing a number of alternative systems including multi-valued, interval, and other types of non-binary logic systems [1-3].

On the other hand, logical paradoxes such as the well-known Barber paradox that was widely discussed in the context of set theory [4] produced important insights into the questions of completeness of mathematical systems [5,6].

The paradox in its common formulation: "in a town, does a barber exist if they shave those who does not shave themselves" can be described by a simple equation on a certain set $X=\{x\}$ for the probability of a relationship $P_{U}(b, x), b$ being a special member of $X$, the barber:

$$
P_{U}(b, x)=1 \text { for } x: \neg\left(P_{U}(x, x)=1\right) ; P_{U}(b, x)=1-P_{U}(x, x)
$$

In the interpretation of conventional binary logic, where $P_{U}$ can only have values 0 or 1 , it follows immediately that the equation is not well defined for any pair $(b, y), b$ $=y$ that is the statement of the paradox.

Evidently, in non-conventional logic systems, the limitation on the values of $P_{U}$ does not apply, in fact, in such systems $P_{U}$ can be a classical or quantum variable with values in a field A, where A can be a discrete set (multi-valued logic, [1]), a continuous range [3] or an abstract algebraic field satisfying certain conditions discussed in Section 2 [7].

The intent of this work is to investigate interpretations and possible solutions of the paradox in different logic systems, both classical and quantum. It is expected that such an investigation can be instrumental in understanding essential questions in completeness of logic systems. 


\section{Non-Binary Logic Systems}

Based on formulation of the paradox statement (1) interpretations of it will be considered in several common non-conventional logic systems. As before it will be assumed that logical variables take value in a field $A$, supporting logical operations of conjunction and disjunction as:

$$
a \wedge b=a \times b ; a \vee b=a+b
$$

$a, b \in A$, and additionally, satisfying the conjunction condition:

$$
a \times b=E \rightarrow a=E ; b=E
$$

where $E$, the unity element (equivalent to the "True" value in conventional binary logic). In any logic system where the paradox can be resolved, a solution to (1), at least one, has to exist for at least one member, $b \in X$ :

$$
P_{U}(b, b)=E-P_{U}(b, b)=P_{U}(b) ; P_{U}(b)+P_{U}(b)=E, P_{U}(b) \neq E
$$

being the necessary and sufficient condition of the resolution of the paradox in the logical system defined by $A$.

\subsection{Multi-valued Logic Systems}

In a multi-valued logic system $L(A)$ with values in a discrete field $A$ of cardinality $N>$ 2 the condition (4) defines the necessary and sufficient condition for the resolution of the paradox in $L$. First, a non-trivial value $m \in A$ has to exist such that $m+m=E$; secondly, there has to exist an element $b \in X$ such that $P_{U}(b)=m$.

$$
\exists m \in A: m+m=E ; \exists b \in X: P_{U}(b)=m
$$

Essentially the condition (5) requires a rationalization extension of the binary field in the Boolean system in a similar way to extension of the natural to rational numbers. From (5) it follows then that a minimal logic system in which the paradox can be resolved has to have at least three values: $0,1, m$.

In such a system a barber with a $P_{U}$ value of $m$ would indeed satisfy the condition (5) and the paradox would have a valid answer: the barber shaves themselves with a probability of $m$.

Finally, if $A$ can have rational values, solving (4) naively yields $m=1 / 2$. It can be interpreted as the third value in the logic system, that of a maximum uncertainty. Adding it in the logic system thus allows to resolve the paradox.

\subsection{Continuous Interval Logic}

In a continuous interval logic system $I(A)$, a logical variable can take values in a continuous interval, commonly $[0,1]$ with values 0,1 representing truth values of binary logic and values $0<p<1$, different degrees of uncertainty [3]. From the perspective of the barber's paradox this case is similar to the case of a multi-valued system $L(A)$ considered in Section 2.1 with $A=I[0,1]$. 
Again, applying (4) one obtains the condition for existence of a barber element in I(A) as:

$$
\exists r \in I: r+r=E ; \exists b \in X: P_{U}(b)=r
$$

If $I=[0,1]$ with standard addition and multiplication algebra, (6) yields $r=1 / 2$ i.e., as in the previous case of a multi-valued logic system, the value of maximum uncertainty.

Consequently, in interval logic systems solution of the paradox is possible with the condition defined by (6).

\subsection{Abstract Algebraic Logic Systems}

The results in Section 2.1 and 2.2 can be readily extended to logic systems with values in abstract field $A$ with logical algebra satisfying certain conditions [7]. The conditions for the existence of a barber element in such systems $L(A)$ would amount to conditions on the cardinality of $A, \operatorname{Card}(A)>2$ and the existence of an "ambiguous element" (at least one) satisfying the condition (5), $m: m+m=E, m \neq E$.

\subsection{Quantum Logic Systems}

In quantum logic [9], a quantum logic variable $Y$ with values in a field $A$ has an associated distribution function $\Psi(p)$ that produces an observed value $y=\|\Psi(p)\|$, $p$ being the parameters of $Y$.

As the act of observation is inherent in a quantum system [9], before investigating the extension of the paradox to quantum logic systems, correct definitions and interpretations should be made. In a quantum system one can interpret classical statements such as "shaves / does not shave themselves" as defined by the outcome of a set of observations $T$ :

$$
\left.P_{U}(x)\right|_{T}=\left.\overline{\left\{p_{1} \ldots p_{N}\right\}}\right|_{T} \mid
$$

where $\left\{p_{k}\right\}$ represent the outcomes of observations of the variable $P_{U}(x)$ on the observation set $T$. One can then interpret statements as "X shaves themselves", "X does not shave themselves" as, respectively: $\left.P_{U}(X)\right|_{\mathrm{T}}>1-\varepsilon,\left.P_{U}(X)\right|_{\mathrm{T}}<\varepsilon$ with $\left.P_{U}(X)\right|_{\mathrm{T}}$ defined by (6).

With the definitions thus obtained, one can interpret Barber's paradox in a quantum logic system $Q(A)$ as:

$$
P_{U}(b, x)=1 \text { for } b: \neg\left(P_{U}(x, x)=1\right)
$$

similarly to (1), however $P_{U}(b)$, representing the probability function according to definition in (6).

In the analysis of the paradox based on (7) the considerations of classical multivalued logic systems defined by $A$ as discussed in Sections 2.1, 2.2 fully apply. It would be interesting therefore, to begin with the case of binary quantum logic where logic variables $P_{U}(x)$ can take values $\{0,1\}$ with a quantum probability function $\Psi(x, p)$. 
The common formulation of the paradox (Section 1) implies that the set of "not shaves", $N S$ is constructed initially based on observation set $T_{N S}$ :

$$
N S:\left.P_{U}(x, x)\right|_{T_{n S}}<\varepsilon
$$

Then, a barber in $X$ will have to shave all elements in NS, so a barber exists if and only if there is an element $b$ in $N S$ that shaves all its members, including itself i.e.:

$$
\exists b \in N S: P_{U}(b, b)=P_{U}(b)>1-\varepsilon
$$

Since the set $N S$ is already defined, the only way to verify the condition in (9) is to conduct a new set of observations $T_{S}$ for elements in $N S$. Then, the existence of a barber element $b$ in a binary quantum logic system $Q_{b}$ is possible if both of the conditions (8) and (9) are satisfied:

$$
\exists b \in N S:\left.P_{U}(x)\right|_{T_{n S}}<\varepsilon ;\left.P_{U}(b)\right|_{T_{S}}>1-\varepsilon
$$

Now one can observe that unlike classical binary logic system where observed value is constant, the condition in (10) is possible to satisfy in a binary quantum logic system with a finite set of observation for any element for which logic variable $\Psi(x, p)$ takes both values $0,1: 0 \leq \Psi(\mathrm{x}, \mathrm{p}) \leq 1$ if the condition is satisfied over sets of observations $T_{N S}, T_{S}$

Consequently, one can conclude that unlike classical binary system, quantum binary logic system does not produce barber's paradox under the identified condition:

$$
\exists b \in X: 0 \leq \Psi(\mathrm{b}, \mathrm{p}) \leq 1
$$

An interesting observation here, reminiscent of the Shrödinger cat paradox [10], is that the existence of the barber in this case is dependent on specific sets of observations $T_{N S}, T_{S}$, and in the same binary quantum logic system $Q_{b}$ a barber may exist or not depending on a specific observation. Implications of this finding are further discussed in the Discussion section.

Finally, from (10) it can be readily obtained that the probability of the existence of the barber element in a binary quantum logic system with the observation sets $T_{N S}, T_{S}$ can be described by a variable $\beta(N)$ dependent on the number of observations, suggesting that statements such as "barber exists" in such logical system themselves can be interpreted as non-binary multi-valued logical variables.

\subsection{Summary}

Following the analysis of the Barber paradox in different non-classical logic systems as discussed in the preceding sections, in this section the obtained results on the existence and conditions of the barber, and the conditions on the logical system necessary for the existence of a solution are summarized. In Table 1, the "Condition" column shows the necessary condition on the logical system for the existence of a barber element, i.e., the existence of a solution to the paradox. 
As can be concluded from these results, Barber paradox can be resolved in all nonclassical logic systems with the conditions discussed in the preceding Sections 2.1 2.4 .

Table 1. Barber's paradox in non-classical logic systems

\begin{tabular}{lccc}
\hline Logic System & Barber's paradox & Condition & Comments \\
\hline Classical binary logic & Exists & N/A & \\
\hline $\begin{array}{l}\text { Multi-valued logic } \\
\text { L(A) }\end{array}$ & Conditional & $m+m=E$ & $\begin{array}{c}\text { Condition defined by } \\
\text { logic algebra A }\end{array}$ \\
\hline $\begin{array}{l}\text { Interval-valued logic } \\
\text { I(A) }\end{array}$ & Conditional & $r+r=E$ & Same as L(A) \\
\hline $\begin{array}{l}\text { Binary Quantum } \\
\text { logic Qb }\end{array}$ & Conditional & observational & $\begin{array}{c}\text { Defined by observation } \\
\text { set }\end{array}$ \\
\hline $\begin{array}{l}\text { Non-binary Quantum } \\
\text { Logic Q(A) }\end{array}$ & Conditional & $\begin{array}{c}\text { L(A) }+ \text { observa- } \\
\text { tional }\end{array}$ & $\begin{array}{c}\text { Defined by logic algebra } \\
\text { A and observation set }\end{array}$ \\
\hline
\end{tabular}

\section{Discussion}

As discussed in the preceding sections, non-classical logic systems provide new logical frameworks to analyze and interpret well-known logical paradoxes. As was shown, one of such paradoxes, the Barber's, can have solutions in non-classical logic systems, including multi-valued, interval and quantum logic systems, that can be interpreted as limitations or incompleteness of classical binary logic systems. As has been demonstrated, extensions to more nuanced systems, specifically, and addition of another value of maximum uncertainty allows to avoid the paradox.

In the analysis of the paradox in non-classical systems, an interesting connection has been observed between formal logic and algebraic structures in multi-valued logic systems. A condition of existence of a solution to the paradox is tied to specific conditions on the algebra of the logical variables that are general for multivalued, interval and abstract algebra-valued logical systems.

Quantum logic systems present another perspective in the analysis of classical logical paradoxes. In a quantum system, definitions that can be self-obvious in classical logic need to be extended to include the essential role of observation. As was shown in Section 2.4, binary quantum logic systems that do not satisfy algebraic conditions of the multi-valued classical logic systems can still have solutions to the paradox that are conditional on specific series of observations.

This finding provides an example of logic systems where paradoxes or statements themselves can have not binary (true or false), but probabilistic, discrete or continuous logical values. For example, as was shown in the analysis in Section 2.3, the statement "barber exists" in such a system can be represented by a continuous logical variable 
$\beta(N)$ representing the probability of existence of a barber element $b$ that is dependent on the number of observations $N$.

This observation of course can be extended to well-known paradoxes in quantum physics, such as Shrödinger cat. Interpreting the statement of paradox as a multi-valued logical variable associated with the probability of observation of a certain outcome removes the appearance of a paradoxical situation that arises from the assumption, and / or expectation that it can be interpreted in terms of classical, binary logic.

It is hoped that further analysis of statements and paradoxes in classical logic in nonbinary logic systems may yield more interesting results on the connections between logical and algebraic systems.

\section{References}

1. Bergmann, M.: An introduction to many-valued and fuzzy logic: semantics, algebras, and derivation systems, Cambridge University Press (2008).

2. Hájek P.: Metamathematics of fuzzy logic. Kluwer (1998).

3. Henson C.W., Iovino J.: Ultraproducts in analysis. In: Analysis and Logic, London Mathematical Society Lecture Note Series 262, Cambridge University Press (2002)

4. Russel B.: The philosophy of logical atomism. Reprinted in The Collected Papers of Bertrand Russell, 1914-19, 8, 228.

5. Herrlich, H.: Axiom of Choice. Lecture Notes in Math, Springer-Verlag (2006).

6. Berto, F.: The Gödel Paradox and Wittgenstein's Reasons. Philosophia Mathematica (III) 17 (2009).

7. Font, J. M.: An abstract algebraic logic view of some multiple-valued logics. In M. Fitting, E. Orlowska (eds.), Beyond two: theory and applications of multiple-valued logic, SpringerVerlag, pp. 25-57 (2003).

8. Birkhoff G. and von Neumann J.: The logic of Quantum Mechanics. Annals of Mathematics, 37, 823-843 (1936).

9. Schlosshauer, M.: Decoherence, the measurement problem, and interpretations of quantum mechanics. Rev. Mod. Phys. 76 (4) 1267-1305 (2005).

10. Schrödinger, E.: Die gegenwärtige Situation in der Quantenmechanik (The present situation in quantum mechanics. Naturwissenschaften, 23 (48) 807-812 (1935). 